\title{
Magnetoresistance of the ferromagnetic nanocontacts fabricated by electrodeposition
}

\author{
CHENG Hao*, YANG Wei, LIU Hong, LI HongLian \& WANG LingJiang \\ School of Electronic and Information Engineering, Chengdu University, Chengdu 610106, China
}

Received January 16, 2012; accepted April 14, 2012; published online August 30, 2012

A " $T$ " shaped micro-gap was fabricated by mechanical polishing between two $\mathrm{Cu}$ film electrodes on the surface of single-sided bonded copper. A nano-gap was then fabricated in the prepared micro-gap by resistance feedback controlled electroplating. Finally $\mathrm{Ni}_{80} \mathrm{Fe}_{20}$ ferromagnetic nanocontacts of several sizes were fabricated in the prepared nano-gap by resistance feedback controlled electroplating. The magnetoresistance of each $\mathrm{Ni}_{80} \mathrm{Fe}_{20}$ ferromagnetic nanocontact was not related to its size. Fabrication of the $\mathrm{Ni}_{80} \mathrm{Fe}_{20}$ ferromagnetic nanocontacts in the nano-gap can reduce the contribution of magnetostriction to the magnetoresistance. The magnetoresistance values of the $\mathrm{Ni}_{80} \mathrm{Fe}_{20}$ ferromagnetic nanocontacts were as high as those of the Ni ferromagnetic nanocontacts. This implies that the contribution of magnetostriction to the ballistic magnetoresistance of the ferromagnetic nanocontacts can be neglected. The ferromagnetic nanocontacts fabricated in this study, and in other cases, have two anisotropic interfaces on the sides of the nanocontacts. However, the magnetic field can alter the contribution of the interaction between the two anisotropic interfaces to the ballistic magnetoresistance of the ferromagnetic nanocontacts, and this effect can not be ruled out yet.

ferromagnetic nanocontacts, resistance feedback controlled electroplating, ballistic magnetoresistance

Citation: Cheng H, Yang W, Liu H, et al. Magnetoresistance of the ferromagnetic nanocontacts fabricated by electrodeposition. Chin Sci Bull, 2013, 58: 598-602, doi: $10.1007 / \mathrm{s} 11434-012-5393-7$

Nano-magnetism was first applied in high density hard disk drives in 1997. Since then it has had a significant impact on daily life and became a research focus [1]. The development of the electronic devices is trending toward ultra-high density, with the microprocessor containing more information units and electrons transported between them at a higher speed. Manufacture of information units with only a few atoms could soon be possible, and these units could be successfully integrated into electronic devices if the developing speed of the electronic devices is continued. However, at a certain scale, transport of the electrons in and between the information units is dominated by the conductance quantization effect, the life time of the units is greatly reduced [2], and the transport behavior of the electrons becomes ballistic [3]. In the past two decades, progress in the nanomagnetism research has been slow because the stability of the information units decreases sharply as the size decreases

*Corresponding author (email: hchengkeke@yahoo.com) and there are few methods to study nano-magnetism. However, with the emergence of spintronics and for manufacture of transistors consisting of a few atoms, the characteristics of the materials at the atomic scale need to be clarified $[4,5]$. For future applications of the ballistic magnetoresistance (BMR) effect in the ultra-high density magnetic storage and magnetic sensors, fabrication of ferromagnetic nanoconstrictions and nanocontacts with giant spin valve effects at room temperature has become a research focus in recent years [6-8].

The BMR effects of ferromagnetic nanocontacts from $70 \%$ to $3000 \%$ [7], and even up to infinity [9], at room temperature have been reported in recent years. However, some experiments have shown that high BMR at room temperature is because of structural changes in the applied magnetic field and not because of a giant spin valve effect $[10,11]$. Many studies focused on the electrical and mechanical characteristics of small nanocontacts, and even those on atomic scale [12-14]. Nanocontacts have a longer 
time to maintain stability at very low temperatures than at higher temperatures. Despite these studies, investigation of the characteristics of these materials at nano and atomic scales remains challenging. Electron beam lithography and vacuum film deposition have been used to fabricate a $20 \mathrm{~nm}$ width thin film ferromagnetic nanoconstriction on silicon [15]. This film is equivalent to 200 atoms (atom $\varnothing 0.1 \mathrm{~nm}$ ) arranged in parallel on the wafer surface [16]. Electronbeam lithography is limited to fabrication of small scale nanocontacts. Electrochemical processes can be performed on the atomic scale, and are reversible and simple, in that they do not require sophisticated equipment. Although electrochemical technology is not compatible with the modern semiconductor industry, it is an important method for studying the characteristics of materials at nano and atomic scales. According to the relationship between the resistance and the size of the ferromagnetic nanocontacts, a ferromagnetic nanocontact of a desired size can be fabricated by resistance feedback controlled electroplating. Molecular electronics predicts that the information units will be limited at the molecular and atomic scale before the future quantum computer age [17]. The assembly, manipulation and characterization techniques for molecular devices are different from those used in the modern semiconductor industry, and electrochemical processes could be used to fabricate a stable conductive channel between the microprocessor and the molecular information units. Recently, to rule out the interference from magnetostriction and the static magnetic force with the BMR of the ferromagnetic nanocontacts, ferromagnetic nanocontacts fabricated by electrodeposition between vacuum deposited thin film nano-gaps on a silicon wafer have been reported $[8,18]$. However, the electrochemical processes are dynamic, and it is difficult to fabricate ferromagnetic nanocontacts by electrodeposition between vacuum deposited thin film nano-gaps because of weak adhesion of this type of film to the silicon. Because the electrodepositing can easily lead to the thin film off from the wafer, it is necessary to find a way to fabricate nano-gaps with stronger adhesion to the substrate.

In this paper, fabrication of $\mathrm{Ni}_{80} \mathrm{Fe}_{20}$ ferromagnetic nanocontacts of different sizes in nano-gaps by resistance feedback controlled electroplating was investigated. A " $\mathrm{T}$ " shaped micro-gap was fabricated by mechanical polishing between two $\mathrm{Cu}$ film electrodes on the surface of singlesided bonded copper. The nano-gap was fabricated in the " $T$ " shaped micro-gap by resistance feedback controlled electroplating. The magnetoresistance of the fabricated $\mathrm{Ni}_{80} \mathrm{Fe}_{20}$ ferromagnetic nanocontacts was studied. The contribution of magnetostriction to the BMR of the ferromagnetic nanocontacts was investigated.

\section{Experimental methods}

First, the "T" shaped micro-gap was fabricated by mechan- ical polishing between two $\mathrm{Cu}$ film electrodes on the surface of single-sided bonded copper. All areas, other than that where the $\mathrm{Ni}_{80} \mathrm{Fe}_{20}$ ferromagnetic nanocontacts was to be fabricated, were covered with the super glue. When the super glue dried, the prepared micro-gap was placed in an electrolyte of $0.5 \mathrm{~mol} / \mathrm{L} \mathrm{Na}_{2} \mathrm{SO}_{4}$ and $0.01 \mathrm{~mol} / \mathrm{L} \mathrm{CuSO}_{4}(\mathrm{pH}$ $6.0)$ as an electrochemical cell cathode. The copper nanogaps were fabricated by electrodeposition of copper on the surfaces of two electrodes by resistance feedback controlled electroplating at $-0.15 \mathrm{~V}$ relative to the saturated calomel electrode. The prepared nano-gap was removed from the electrochemical cell and cleaned with distilled water. It was then placed in an electrolyte of $0.2 \mathrm{~mol} / \mathrm{L} \mathrm{NiSO}_{4}, 0.01$ $\mathrm{mol} / \mathrm{L} \mathrm{FeCl}_{2}, 0.4 \mathrm{~mol} / \mathrm{L} \mathrm{H}_{3} \mathrm{BO}_{3}$ and $0.2 \mathrm{~g} / \mathrm{L}$ sodium dodecyl sulfate $(\mathrm{pH}$ 3.0) [19] as an electrochemical cell cathode to fabricate $\mathrm{Ni}_{80} \mathrm{Fe}_{20}$ ferromagnetic nanocontacts of different sizes. $\mathrm{Ni}_{80} \mathrm{Fe}_{20}$ was electrodeposited by resistance feedback controlled electroplating at $-1 \mathrm{~V}$ relative to the saturated calomel electrode. All the electrochemical processes were automatically controlled by LabView software (National Instruments, Austin, TX). Scanning electron microscopy (SEM) was used to characterize the width of the $\mathrm{Cu}$ nano-gap and the morphology of the $\mathrm{Ni}_{80} \mathrm{Fe}_{20}$ ferromagnetic nanocontacts. The magnetoresistance of the $\mathrm{Ni}_{80} \mathrm{Fe}_{20}$ ferromagnetic nanocontacts was measured in-situ at room temperature with a measurement system controlled by LabView software.

\section{Results and discussion}

A $20 \mu \mathrm{m}$ gap was observed for the "T" shaped micro-gap (Figure 1) by the optical microscopy. Adhesion of the prepared " $T$ " shaped micro-gap to the substrate was much stronger than that of a vacuum deposited thin film on a wafer. The issue with a micro-gap prepared in this way is that the copper is $20 \mu \mathrm{m}$ thick. Figure 2 shows the resistance feedback controlled electroplating process [20]. In this figure, the working electrodes $\left(\mathrm{W}_{1}\right.$ and $\left.\mathrm{W}_{2}\right)$ are the two copper film electrodes, CE is the Pt counter electrode, and REF is the saturated calomel electrode. The nano-gap and the ferromagnetic nanocontacts can be fabricated between the two

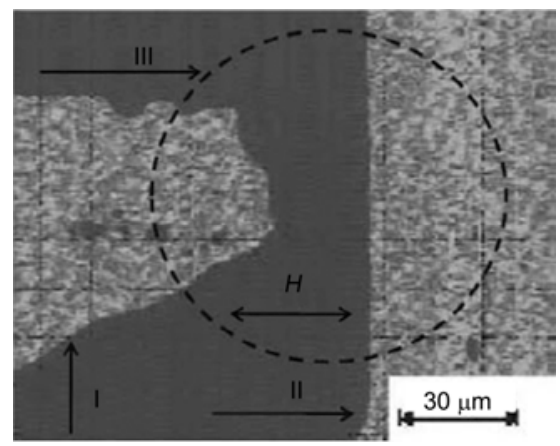

Figure 1 Optical microscopy image of the morphology of a prepared " $T$ " shaped micro-gap. 


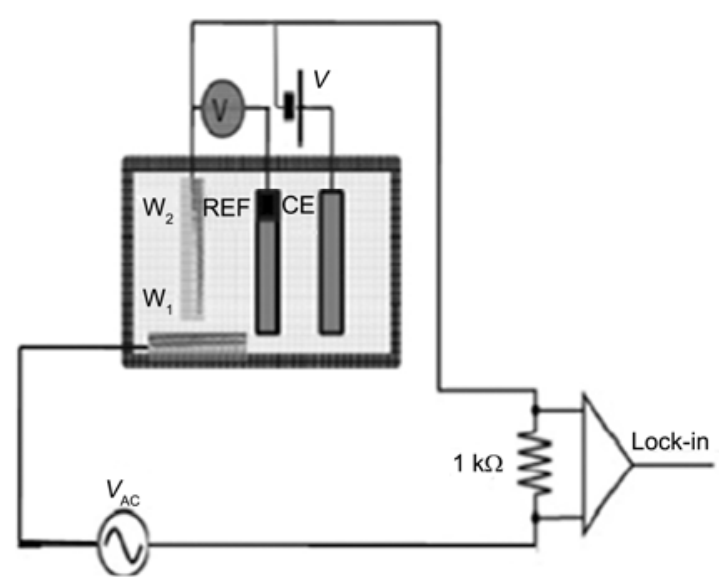

Figure 2 Schematic diagram of the resistance feedback controlled electroplating process.

working electrodes $\left(\mathrm{W}_{1}\right.$ and $\left.\mathrm{W}_{2}\right)$ by an electrochemical process. The width of the nano-gap and the size of the ferromagnetic nanocontacts can be controlled by changing the resistance between $\mathrm{W}_{1}$ and $\mathrm{W}_{2}$. The relationship between the width of the gap and the resistance between $\mathrm{W}_{1}$ and $\mathrm{W}_{2}$ measured in the electrolyte is important for fabrication of the nano-gaps with desired widths. We found that as the surface areas of the copper electrodes increased, the width of the gap and the resistance between $\mathrm{W}_{1}$ and $\mathrm{W}_{2}$ decreased. Initially, the resistance between the $\mathrm{W}_{1}$ and $\mathrm{W}_{2}$ decreased gradually. Then, when the width of the gap was at the nanoscale, a sudden drop of the resistance between the $\mathrm{W}_{1}$ and $\mathrm{W}_{2}$ was observed. At this point, electrodeposition of copper should be stopped immediately. The widths of the nanogaps fabricated in this way are typically between 100 and $200 \mathrm{~nm}$. The widths of the nanogaps do not need to be measured directly by SEM, as the sudden drop in resistance indicates that the gap will be nanoscale. Figure 3 shows the typical morphology of the prepared copper nano-gaps. A $150 \mathrm{~nm}$ nano-gap can be clearly seen in this figure. The resistance and the size of the ferromagnetic nanocontacts can be

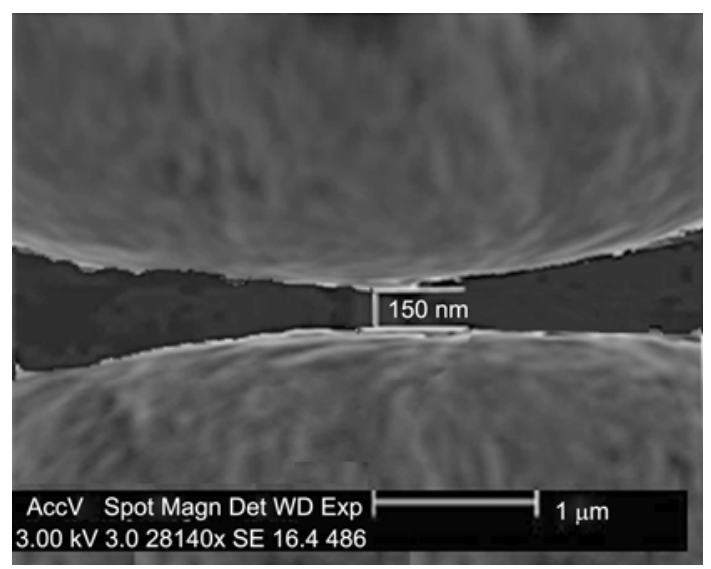

Figure 3 SEM image of the morphology of a prepared nano-gap. related by the following equation, $d=\sqrt{1000 / R(\Omega)}$ (nm) [21], where $d$ is the diameter and $R$ is the resistance of the ferromagnetic nanocontacts. Comparison of the default resistance and the actual resistance, $\mathrm{Ni}_{80} \mathrm{Fe}_{20}$ ferromagnetic nanocontacts of a desired size can be fabricated by resistance feedback controlled electroplating. It should be noted that the default resistance and the actual resistance are only equal in theory for the limitation of the feedback control. There must be an error between the default resistance and the actual resistance, to reduce the time for the fabrication of the $\mathrm{Ni}_{80} \mathrm{Fe}_{20}$ ferromagnetic nanocontacts, and the error was set to $5 \Omega$. If we want to fabricate an $R$ ferromagnetic nanocontact, when the actual resistance of the ferromagnetic nanocontact is in the range of $R \pm 5 \Omega$, the electrochemical process should be stopped and the ferromagnetic nanocontact of the desired size will be obtained. There are several methods to measure the resistance between the two electrodes during fabrication of the nano-gap and ferromagnetic nanocontacts by resistance feedback controlled electroplating. These methods include $50 \%$ duty cycle periodic current pulse [22] and real-time resistance measurements [23]. For this study real-time resistance measurement was used to measure the resistance between the two electrodes. A millivolt AC voltage source was selected to reduce the impact of measuring the resistance on the electrochemical process. The frequency of the AC voltage source was controlled at below $10 \mathrm{~Hz}$ to reduce the effect of interfacial capacitance between the two electrodes. The resistance between the two electrodes was measured directly because of the large range of change in the resistance between the two electrodes during fabrication of the nano-gap. The resistance of the $\mathrm{Ni}_{80} \mathrm{Fe}_{20}$ ferromagnetic nanocontacts was measured by measuring the voltage changes of a $1 \mathrm{k} \Omega$ resistor in series with the nanocontacts. Figure 4 shows the morphology of the $\mathrm{Ni}_{80} \mathrm{Fe}_{20}$ ferromagnetic nanocontacts fabricated by electrodeposition. Although the position and size of the nanocontact can not be accurately indentified from the figure, the resistance between the two electrodes

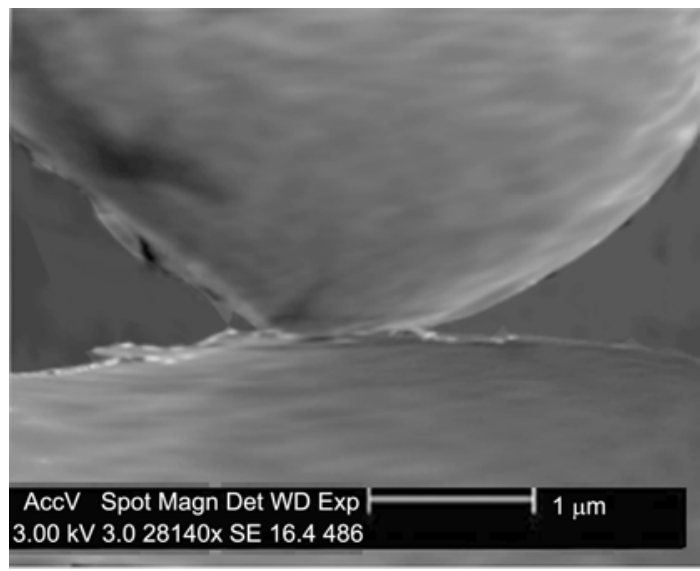

Figure 4 SEM image of the morphology of a prepared $\mathrm{Ni}_{80} \mathrm{Fe}_{20}$ nanocontact. 
indicates that a nanocontact exists between the electrodes. The size of the nanocontact can be calculated using $d=\sqrt{1000 / R(\Omega)}$. Figure 5 shows the magnetoresistance of a prepared $\mathrm{Ni}_{80} \mathrm{Fe}_{20}$ ferromagnetic nanocontact measured at room temperature with the magnetic field applied in the same plane as the current. The diameter of this nanocontact was $5.2 \mathrm{~nm}$, and the magnetoresistance was about $90 \%$. The magnetoresistance values of the $\mathrm{Ni}_{80} \mathrm{Fe}_{20}$ ferromagnetic nanocontacts with the resistance values between $7 \Omega$ and $36.5 \Omega$ at room temperature are shown in Figure 6. The corresponding diameters of the ferromagnetic nanocontacts were 5.2, 5.6, 6.6, 8.2 and $12.1 \mathrm{~nm}$. As shown in this figure, the magnetoresistance was independent of the size of the ferromagnetic nanocontacts. This means that, in addition to the size of the nanocontact, its magnetoresistance is also affected by other factors. The Ni ferromagnetic nanocontacts fabricated by mechanical and electrodeposition methods with magnetoresistance values $>70 \%$ at room temperature have been reported [6,7,22]. The magnetostriction of the $\mathrm{Ni}_{80} \mathrm{Fe}_{20}$ ferromagnetic nanocontacts fabricated in the nano-gap was much smaller than that of the reported $\mathrm{Ni}$ ferromagnetic nanocontacts, and the magnetoresistances of the $\mathrm{Ni}_{80} \mathrm{Fe}_{20}$ ferromagnetic nanocontacts was as high as that of the reported $\mathrm{Ni}$ ferromagnetic nanocontacts. This means that the effect of magnetostriction on the BMR of the ferromagnetic nanocontacts can be neglected. However, both the $\mathrm{Ni}_{80} \mathrm{Fe}_{20}$ ferromagnetic nanocontacts fabricated in the nano-gaps and the $\mathrm{Ni}$ ferromagnetic nanocontacts fabricated by other methods are formed by two anisotropic interfaces. The applied magnetic field alters the interaction between the two anisotropic interfaces, and the contribution of this change to the BMR of the ferromagnetic nanocontacts can not be ruled out yet.

\section{Conclusion}

In conclusion, nano-gaps between two electrodes and

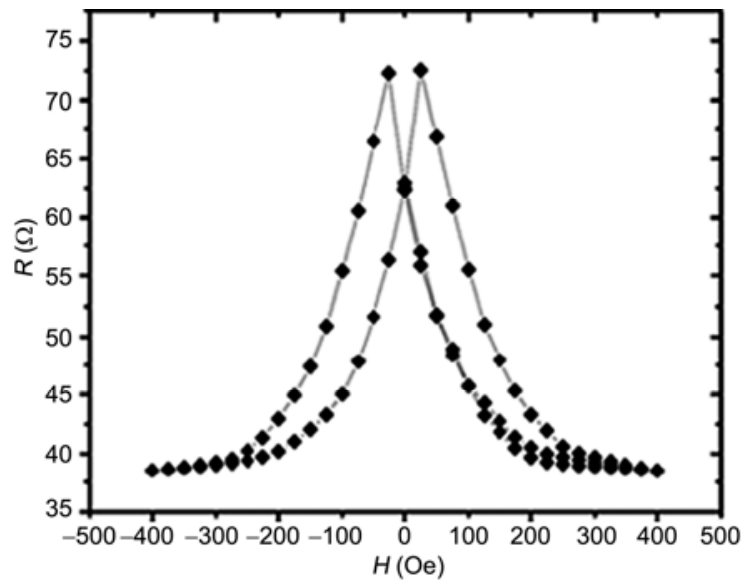

Figure 5 The typical magnetoresistance of the prepared $\mathrm{Ni}_{80} \mathrm{Fe}_{20}$ ferromagnetic nanocontacts measured at room temperature.

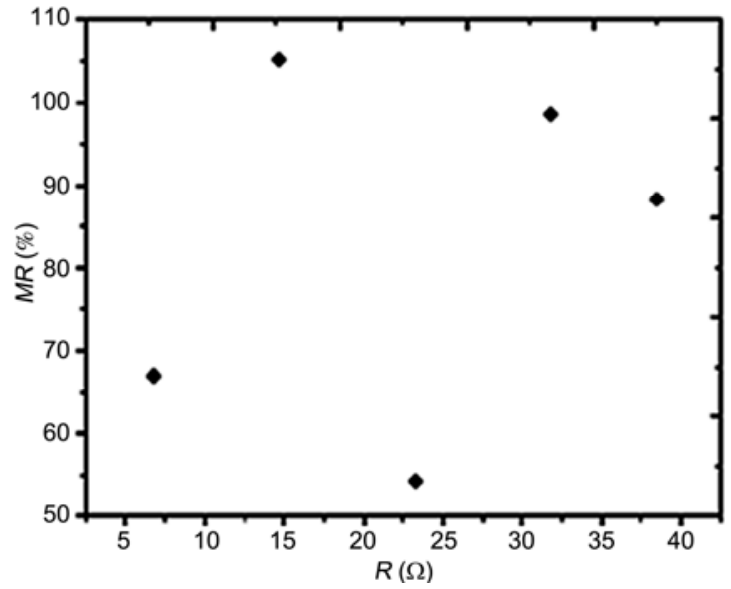

Figure 6 The magnetoresistance values of the prepared $\mathrm{Ni}_{80} \mathrm{Fe}_{20}$ ferromagnetic nanocontacts of different resistance measured at room temperature.

$\mathrm{Ni}_{80} \mathrm{Fe}_{20}$ ferromagnetic nanocontacts were fabricated by resistance feedback controlled electroplating. The magnetostriction of the prepared $\mathrm{Ni}_{80} \mathrm{Fe}_{20}$ ferromagnetic nanocontacts was much smaller than that of reported Ni ferromagnetic nanocontacts. The magnetoresistance of the prepared $\mathrm{Ni}_{80} \mathrm{Fe}_{20}$ ferromagnetic nanocontacts was as high as that of reported Ni ferromagnetic nanocontacts. These experiments show that the contribution of magnetostriction to the BMR of the ferromagnetic nanocontacts can be neglected. However the magnetic field can alter the interaction between the two anisotropic interfaces, and the contribution of this to the BMR of the ferromagnetic nanocontacts cannot be ruled out by these experiments.

1 Grünberg P. From spinwaves to giant magnetoresistance (gmr) and beyond. Nobel Lecture, December 8, 2007

2 Schnable G L, Keen R S. Failure mechanisms in large-scale integrated circuits. IEEE Trans Electr Devices, 1969, 4: 322-332

3 Landauer R. Electrical resistance of disordered one-dimensional lattices. Philos Mag, 1970, 21: 863-867

4 Wolf S A, Awschalom D D, Buhrman R A, et al. Spintronics: A spin-based electronics vision for the future. Science, 2001, 294: 1488-1495

5 Aviram A, Ratner M A. Molecular rectifiers. Chem Phys Lett, 1974, 29: 277-283

6 Garcia N, Munoz M, Zhao Y W. Magnetoresistance in Ni nanocontacts in excess of $200 \%$ at room temperature and 100 Oe field. Phys Rev Lett, 1999, 82: 2923-2926

7 Chopra H D, Hua S Z. Ballistic magnetoresistance over $3000 \%$ in Ni nanocontacts at room temperature. Phys Rev B, 2002, 66: 020403

8 Chopra H D, Matthew R S, Jason N A, et al. The quantum spin-valve in cobalt atomic point contacts. Nat Mater, 2005, 4: 832-837

9 Hua S Z, Chopra H D. 100000\% ballistic magnetoresistance in stable Ni nanocontacts at room temperature. Phys Rev B, 2003, 67: 060401

10 Haug T, Perzlmaier K, Back C H. In situ magnetoresistance measurements of ferromagnetic nanocontacts in the Lorentz transmission electron microscope. Phys Rev B, 2009, 79: 024414

11 Egelhoff W F Jr, Gana L, Ettedguia H, et al. Artifacts that mimic ballistic magnetoresistance. J Magn Magn Mater, 2005, 287: 496-500

12 Champagne A R, Pasupathy A N, Ralph D C. Mechanically adjustable and electrically gated single-molecule transistors. Nano Lett, 
2005, 5: 305-308

13 Rubio-Bollinger G, Bahn S R, Agraït N, et al. Mechanical properties and formation mechanisms of a wire of single gold atoms. Phys Rev Lett, 2001, 87: 026101

14 Scheer E, Agraït N, Cuevas J C, et al. The signature of chemical valence in the electrical conduction through a single-atom contact. Nature, 1998, 394: 154-157

15 Cheng H, Yang W, Liu H, et al. Magnetoresistance of the thin film ferromagnetic nanoconstriction. Chin Sci Bull, 2012, 57: 445-449

16 Agraït N, Yeyati A L, van Ruitenbeek J M. Quantum properties of atomic-sized conductors. Phys Rep, 2003, 377: 81-279

17 Reed M A, Zhou C, Muller C J, et al. Conductance of a molecular junction. Science, 1997, 278: 252-254

18 Sokolov A, Zhang C J, Tsymbal E Y, et al. Quantized magnetoresistance in atomic-size contacts. Nat Nanotech, 2007, 2: 171-175
19 Steven D L, Shirley R, Daniel T S. Characterization of $\mathrm{Ni}_{x} \mathrm{Fe}_{1-x}(0.10$ $<x<0.95)$ electrodeposition from a family of sulfamate-chloride electrolytes. J Electrochem Soc, 1999, 4: 1431-1435

20 Shu C, Li C Z, He H X, et al. Fractional conductance quantization in metallic nanoconstrictions under electrochemical potential control. Phys Rev Lett, 2000, 84: 5196-5199

21 Garcia N, Munoz M, Qian G G, et al. Ballistic magnetoresistance in a magnetic nanometer sized contact: An effective gate for spintronics. Appl Phys Lett, 2001, 79: 4550-4552

$22 \mathrm{Li} \mathrm{C} \mathrm{Z,} \mathrm{He} \mathrm{H} \mathrm{X,} \mathrm{Tao} \mathrm{N} \mathrm{J.} \mathrm{Quantized} \mathrm{tunneling} \mathrm{current} \mathrm{in} \mathrm{the} \mathrm{metallic}$ nanogaps formed by electrodeposition and etching. Appl Phys Lett, 2000, 77: 3995-3997

23 Snow E S, Campbell P M. AFM fabrication of sub-10-nanometer metal-oxide devices with in situ control of electrical properties. Science, 1995, 270: 1639-1641

Open Access This article is distributed under the terms of the Creative Commons Attribution License which permits any use, distribution, and reproduction in any medium, provided the original author(s) and source are credited. 